\title{
A THEOREM ON HOLONOMY
}

\author{
BY \\ W. AMBROSE AND I. M. SINGER
}

Introduction. The object of this paper is to prove Theorem 2 of $\S 2$, which shows, for any connexion, how the curvature form generates the holonomy group. We believe this is an extension of a theorem stated without proof by E. Cartan [2, p. 4]. This theorem was proved after we had been informed of an unpublished related theorem of Chevalley and Koszul. We are indebted to S. S. Chern for many discussions of matters considered here.

In $\S 1$ we give an exposition of some needed facts about connexions; this exposition is derived largely from an exposition of Chern [5] and partly from expositions of H. Cartan [3] and Ehresmann [7]. We believe this exposition does however contain one new element, namely Lemma 1 of $\$ 1$ and its use in passing from H. Cartan's definition of a connexion (the definition given in $\$ 1$ ) to E. Cartan's structural equation.

1. Basic concepts. We begin with some notions and terminology to be used throughout this paper. The term "differentiable" will always mean what is usually called "of class $\mathrm{C}^{\infty}$." We follow Chevalley [6] in general for the definition of tangent vector, differential, etc. but with the obvious changes needed for the differentiable (rather than analytic) case. However if $\phi$ is a differentiable mapping we use $\phi$ again instead of $d \phi$ and $\delta \phi$ for the induced mappings of tangent vectors and differentials. If $M$ is a manifold, by which we shall always mean a differentiable manifold but which is not assumed connected, and $m \in M$, we denote the tangent space to $M$ at $m$ by $M_{m}$. If $W$ is a vector field at $M$ we denote its value at $m$ by $W(m)$, and if $x_{1}, \cdots, x_{k}$ is a coordinate system of $M$ we always write $X^{1}, \cdots, X^{k}$ for the corresponding partial derivatives, i.e. $X^{i}=\partial / \partial x_{i}$. We use the word "diffeo" for a $1: 1$ differentiable mapping of one manifold onto another whose inverse is also differentiable and call the manifolds diffemorphic. If $W, W^{\prime}$ are vector fields we write $\left[W, W^{\prime}\right]$ for $W W^{\prime}-W^{\prime} W$ (opposite to [6]). $R^{k}$ will always denote $k$-dimensional Euclidean space of all $k$-tuples of real numbers and $\delta_{1}, \cdots, \delta_{k}$ will always denote the canonical unit elements there, i.e. $\delta_{j}$ $=\left(\delta_{1 j}, \delta_{2 j}, \cdots, \delta_{n j}\right)$.

Let $G$ be a Lie group (i.e. a differentiable group) acting differentiably and effectively on a manifold $F$. If $f \in F$ and $g \in G$ we write $g f$ for the image of $f$ under the action of $g \in G$ and if $t$ is a tangent vector at $f$ we write $g t$ for the image of $t$ (which will be a tangent vector of $g f$ ) under $g$. If $O$ is a manifold we denote by $(O, F, G)$ the family of all transformations of $O \times F \rightarrow O \times F$ of the form : $(o, f) \rightarrow(o, t(o, f))$ when $t$ is any differentiable mapping of $O \times F \rightarrow F$

Received by the editors September 22, 1952. 
such that, for each $o \in O$, the transformation: $f \rightarrow t(o, f)$ coincides with the action of some $g \in G$.

Definition. A bundle is a collection $(B, M, F, G, \pi, \phi)$ where $B, M, F$ are manifolds, $G$ is a Lie group acting differentiably and effectively on $F, \pi$ is a differentiable mapping of $B$ onto $M$, and $\phi$ is a collection of mappings. It is further assumed that

(1) each $\phi \in \Phi$ is a diffeo of some $O \times F$, where $O$ is an open submanifold of $M$, onto $\pi^{-1}(O)$,

(2) $(\pi \circ \phi)(o, f)=o$ for all $(o, f) \in O \times F$,

(3) if $\phi, \psi \in \Phi, \phi: O \times F \rightarrow B, \psi: P \times F \rightarrow B, 0 \# P\left({ }^{1}\right)$, then $\left(\phi^{-1} \circ \psi\right) \mid((0 \cap P)$ $\times F) \in(0 \cap P, F, G)$,

(4) the domains of the $\phi$ in $\Phi$ cover $B$,

(5) the family $\Phi$ is maximal with respect to (1), (2), (3).

Then $B$ is called the bundle space, $M$ the base space, $G$ the group, $\pi$ the projection, $\Phi$ the family of strip maps of the bundle. In the special case where $F$ and $G$ are the same manifold and $G$ acts as itself by left translation, the bundle is called a principal bundle and denoted by $(B, M, G, \pi, \Phi)$.

If $\phi$ is a strip map taking $0 \times F \rightarrow B$, then for each $m \in O$ we define $\phi_{m}: F$ $\rightarrow B$, by $\phi_{m}(f)=\phi(m, f)$. If $\phi, \psi$ are strip maps and $m \in M$ is such that both $\phi_{m}$ and $\psi_{m}$ are defined, then the definition of $(O, F, G)$ and (3) show $\phi_{m}^{-1} \circ \Psi_{m}$ coincides with the action of some $g \in G$ on $F$ and in the case of a principal bundle this means that $\phi_{m}^{-1} \circ \psi_{m}$ is a left translation by some $g \in G$.

Definition. A tangent vector $t$ at a point in a bundle is vertical $\mathrm{ff}\left({ }^{2}\right)$ $\pi t=0$. The linear space of vertical vectors at $b \in B$ will be denoted by $V_{b}$.

A vector field $A$ in the fiber $F$ which is invariant under the action of $G$ gives rise, in a way we shall now describe, to a vertical vector field $Q$ on $B$ (by a vertical vector field we mean one that assigns a vertical vector at each point). To define $Q(b)$ consider any strip map $\phi: O \times F \rightarrow B$, such that $b$ $\in \phi(O \times F)$. If $\phi_{m}(f)=b$ we define $Q(b)=\phi_{m} A(f)$. This definition is independent of the strip map $\phi$ because if $\psi$ is another such strip map then $\phi_{m}^{-1} \circ \psi_{m}$ agrees with the action of some $g \in G$. Hence $\psi_{m}(f)=\phi_{m}(g f)$ for all $f \in F$ and then, using the invariance of $A$, we have $\psi_{m} A(f)=\phi_{m} g A(f)=\phi_{m} A(g f)$. If $A$ is differentiable, then so is $W$ and the mapping thus defined, taking $A \rightarrow W$, preserves brackets (these properties are immediate from the fact that the strip maps $\phi$ are diffeos). In particular, if the bundle is a principal bundle, the invariant vector fields on the fiber are the left invariant vector fields of the group $G$ and these constitute the Lie algebra $L$ of $G$. Hence in this case the mapping taking $A \rightarrow W$ is an iso $\left.{ }^{3}\right)$ of the Lie algebra $L$ of $G$ onto a Lie algebra of vertical vector fields on $B$. Throughout this paper we shall,

(1) $0 \# P$ means 0 and $P$ have a non-null intersection. Similarly $0 \| P$ will mean 0 and $P$ have a null intersection.

(2) ff means: if and only if.

${ }^{(3)}$ We shall omit the appendage "morphism" from an obvious maximal set of words. 
in the case of a principal bundle (and principal bundles will be the only ones we shall seriously consider), reserve the letter $q$ for this iso and the letter 2 for its image $q(L)$. If $t$ is a vertical vector at $b \in B$, then it is trivial that there exists a unique $Q \in 2$ such that $Q(b)=t$; we then say $Q$ is generated by $t$ and if $Q=q(A)$ we also say $A$ is generated by $t$.

If $(B, M, G, \pi, \Phi)$ is a principal bundle, then $G$ can be made to act on $B$ in a natural way that is called right translation. This differentiable and effective action of $G$ on $B$ is defined as follows. If $b \in B, g \in G, \phi$ any strip map, $\phi: O \times F \rightarrow B, b \in \phi(O \times F)$, then write $b=\phi(m, h)$ and define $b g=\phi(m, h g)$. This definition of $b g$ is independent of the choice of the strip map $\phi$ because, for any other strip map $\psi, \phi_{m}^{-1} \circ \psi_{m}$ is a left translation on $G$ and left translations commute with right translations on $G$. The right translation action of $G$ on $B$ is the mapping of $B \times G \rightarrow B:(b, g) \rightarrow b g$. The mapping of $B \rightarrow B$ taking $b \rightarrow b g$ (for any fixed $g \in G$ ) will be denoted by $R_{g}$ and right translation by $g$ on $G$ will also be denoted by $R_{g}$; left translation by $g$ on $G$ will be denoted by $L_{g}$. If $\phi$ is any strip map of $B$, then clearly $\phi_{m} \circ R_{g}=R_{g} \circ \phi_{m}$.

We denote the inner auto $\left.{ }^{3}\right)$ of $G$, corresponding to $g \in G$, by $\alpha(g)$, i.e. $\alpha(g) h=g h g^{-1}$ for $h \in G$, and if $A \in L$ ( $L$ the Lie algebra of $G$ ) we write $\alpha(g) A$ for the element of $L$ defined by: $(\alpha(g) A)(e)=\alpha(g) A(e) . \alpha(g) A$ is sometimes denoted by $a d g A$. For $Q \in 2$ we define $\alpha(g) Q \in 2$ by $\alpha(g) Q=q(\alpha(g) A)$, when $Q=q(A)$. Then $R_{g} Q=\alpha\left(g^{-1}\right) Q$, for if $\phi$ is any strip map of $B$ and $\phi(h)=b$ then $\left(\alpha\left(g^{-1}\right) Q\right)(b)=\phi\left(\left(\alpha\left(g^{-1}\right) A\right)(h)\right)=\phi\left(L_{h} \circ R_{g} \circ L_{g}^{-1} A(e)\right)=\phi\left(R_{g} \circ L_{h g^{-1}} A(e)\right)$ $=\phi\left(R_{g} A\left(h g^{-1}\right)\right)=\left(\phi \circ R_{g}\right) A_{\left(h g^{-1}\right)}=\left(R_{g} \circ \phi\left(A\left(h g^{-1}\right)=R_{g} Q\left(h g^{-1}\right)\right.\right.$.

Some principal bundles of special interest are the bundle of bases $E(M)$ over any manifold $M$ and the bundle of frames $F(M)$ over a Riemannian $M$. We now define $E(M)$. The points of $E(M)$ are all the ordered $(d+1)$-tuples $\left(m, e_{1}, \cdots, e_{d}\right)$ where $m \in M$ and $\left(e_{1}, \cdots, e_{d}\right)$ is any base of $M_{m}$. The projection $\pi$ of $E(M)$ onto $M$ is defined by $\pi\left(m, e_{1}, \cdots, e_{d}\right)=m$. The group (and fiber) $G$ is the full linear group over the reals, i.e. the group of all nonsingular linear transformations of $R^{d}$ onto itself. With each point $b$ of $E(M)$ we can associate an iso $I_{b}$ of $M_{m}$ onto $R^{d}$ by writing $b=\left(m, e_{1}, \cdots, e_{d}\right)$ and defining $I_{b}$ to be the linear transformation taking $e_{i} \rightarrow \delta_{i}$ (for all $i$ ); and conversely, so the points of $E(M)$ could have been defined as the pairs $(m, I)$ where $I$ is such an iso. In this example it is convenient to define the action of $G$ on the bundle before defining the bundle structure. We do this by: if $b$ $=\left(m, e_{1}, \cdots, e_{d}\right)$, then $b g=\left(m, I_{b}^{-1} g I_{b} e_{1}, \cdots, I_{b}^{-1} g I_{b} e_{d}\right)$; this implies that $I_{b g}$ takes $\left(I_{b}^{-1} \circ g \circ I_{b}\right) e_{i} \rightarrow f_{i}$, hence $I_{b g} \circ I_{b}^{-1} \circ g \circ I_{b}=I_{b}$ from which it is clear that $I_{b g}=g^{-1} \circ I_{b}$ (a fact that will be useful later). For any coordinate system $x_{1}, \cdots, x_{d}$ of $M$ with domain $O$ we define $\phi: O \times G \rightarrow E(M):(m, g)$ $\rightarrow R_{g}\left(m, X^{\prime}(m), \cdots, X^{d}(m)\right)\left({ }^{4}\right)$ (now writing $R_{g} b$ for the $b g$ just defined). We introduce coordinates $y_{1}, \cdots, y_{d}, y_{11}, \cdots, y_{d d}$ of $E(M)$, with domain $\pi^{-1}(O)$, by

(4) The notation $f: X \rightarrow Y: x \rightarrow y$ means $f$ is a function from $X$ to $Y$ taking $x$ into $y$. 


$$
y_{j}=x_{j} \circ \phi, \quad e_{j}=\sum_{i} y_{i j}\left(m, e_{1}, \cdots, e_{d}\right) X^{i}(m) .
$$

Such coordinate systems of $E(M)$ obtained from different coordinate systems of $M$ are easily seen to be differentiably related. Since their domains cover $E(M)$ they define a unique differentiable structure on $E(M)$, and this defines $E(M)$ as a manifold. Now let $x_{1}, \cdots, x_{d}$ and $x_{1}^{\prime}, \cdots, x_{d}^{\prime}$ be any two coordinate systems of $M$ with domains $O$ and $O^{\prime}$, let $\phi$ and $\phi^{\prime}$ be the mappings they define as above, and we shall show $\left(\phi^{-1} \circ \phi^{\prime}\right) \mid\left(\left(O \cap O^{\prime}\right) \times G\right)$ is in $\left(O \cap O^{\prime}, G, G\right)$. If $m \in O \cap O^{\prime}, b=\phi(m, e)=\left(m, X^{\prime}(m), \cdots, X^{d}(m)\right), b^{\prime}$ $=\phi^{\prime}(m, e)=\left(m, X^{\prime 1}(m), \cdots, X^{\prime d}(m)\right)$, then $\phi_{m}^{-1} \phi_{m}^{\prime}$ takes $g \in G$ into the $h \in G$ such that $I_{b}^{-1} g I_{b} X^{i}(m)=I_{b^{\prime}}^{-1} h I_{b^{\prime}} X^{1 i}(m)$, i.e. $g$ goes into the $h$ such that $I_{b}^{-1} g \delta_{i}=I_{b^{\prime}}^{-1} h \delta_{i}$ (for all $i$ ). Hence $h=I_{b^{\prime}} \circ I_{b}^{-1} \circ g$, showing that $\phi_{m}^{-1} \circ \phi_{m}^{\prime}$ is left translation by $k=I_{b}, \circ I_{b}^{-1}$ on $G$. And from this expression for $k$ it is easily seen that $k$ is a differentiable function of $m$, thus $\left(\phi^{-1} \circ \phi^{\prime}\right) \mid\left(\left(O \cap O^{\prime}\right) \times G\right)$ is in $\left(O \cap O^{\prime}, G, G\right)$. We define $\Phi$, the family of strip maps of this bundle, to be all $\phi$ obtained from coordinate systems of $M$ as above plus all other mappings $\psi$ of any $P \times G \rightarrow E(M)$ which satisfy conditions (1) and (2) in the definition of a bundle and the condition that $\left(\psi^{-1} \circ \phi\right) \mid((O \cap P) \times G)$ is in $(O \cap P, G, G)$ for such $\phi$ as above. Clearly the $R_{g}$ defined by this bundle structure agrees with the $R_{g}$ used above.

The bundle $F(M)$ is defined similarly, the points in this case being of the form $\left(m, e_{1}, \cdots, e_{d}\right)$ where $m \in M$ and the $e_{i}$ are any orthonormal base of $M_{m} . F(M)$ is in a natural way a submanifold of $E(M)$.

Definition. A connexion $H$ on a principal bundle $\mathbb{B}=(B, M, G, \pi, \Phi)$ is a mapping which assigns to each $b \in B$ a linear subspace $H_{b}$ of $B_{b}$ such that

(1) $H_{b}$ is a linear complement to $V_{b}$; elements of $H_{b}$ will be called horizontal vectors.

(2) $H$ is invariant under the action of $G$ on $B$, i.e. $H_{b g}=R_{g} H_{b}$,

(3) $H_{b}$ depends differentiably on $b$, i.e. if $W$ is any differentiable vector field on $B, H W$ its horizontal part (i.e. $H W$ is the vector field which assigns at each $b \in B$ the projection of $W(b)$ into $\left.H_{b}\right), H W$ is differentiable.

Notation. If $W$ is any vector field on such a $B$ with a connexion $H$ we shall always write $H W$ and $V W$ for the horizontal and vertical parts of $W$; differentiability of $W$ trivially implies that of $V W$.

If $H$ is a connexion on $B$ then, for each $b \in B, \pi \mid H_{b}$ is an iso of $H_{b}$ onto $M_{m}$, where $m=\pi b$. This is immediate from: (1) $\pi$ maps $B_{b}$ onto $M_{m}$, (2) $\pi V_{b}=0$, (3) $\operatorname{dim} H_{b}=\operatorname{dim} M_{m}$.

Definition. If $H$ is a connexion on $B$ and $b \in B$, the holonomy group of $H$ attached to $b$, denoted by $G(b)$, is the set of all $g \in G$ such that $b g$ can be joined to $b$ by a piecewise-differentiable $\left(^{5}\right)$ horizontal curve in $B$. The null holonomy

(5) By a piecewise differentiable curve we mean a curve which is differentiable and has a nonzero tangent vector except at a finite number of points. By horizonal we mean that the tangent vector is in $H_{b}$ at all points $b$ on the curve for which the tangent vector exists. 
group of $H$ attached to $b$, denoted by $G_{0}(b)$, is the set of all $g \in G$ such that $b g$ can be joined to $b$ by a piecewise differentiable horizontal curve in $B$ whose projection into $M$ is null-homotopic.

It is trivial that $G(b)$ and $G_{0}(b)$ are subgroups of $G$ and that if $c=b g$ then $G(c)=g^{-1} G(b) g, G_{0}(c)=g^{-1} G_{0}(b) g$.

If $H$ is a connexion on $E(M)$, then the holonomy group is related to the notion of parallel translation along curves in $M$ so we briefly indicate here how parallel translation is derived from the above concept of a connexion. Let $H$ be a connexion on $E(M)$. If $\rho$ is any piece-wise differentiable curve in $M$, from $m$ to $m^{\prime}$, we shall define a linear transformation $T$ of $M_{m}$ onto $M_{m^{\prime}}$ and this linear transformation is called parallel translation along $\rho$. To define $T$ let $\sigma$ be any horizontal curve in $E(M)$ lying over $\rho$ (i.e. $\rho=\pi \circ \sigma$ ). Then $\sigma$ starts at some point $\left(m, e_{1}, \cdots, e_{d}\right)$ and ends at some point $\left(m^{\prime}, e_{1}^{\prime}, \cdots, e_{d}^{\prime}\right)$. We define $T$ to be the unique linear transformation taking $e_{i} \rightarrow e_{1}^{\prime}$ (for all $i$ ). The horizontal curve $\sigma$ lying over $\rho$ is not unique, but because of the invariance of $H$ under the action of $G$ on $E(M)$ it is easily seen that $T$ is independent of the choice of $\sigma$. The existence of a horizontal curve $\sigma$ lying over $\rho$ can be shown as follows (note that this construction is valid for any connexion, not necessarily on an $E(M)$ ). Consider any curve $\tau$ lying over $\rho$ in $B$-and such curves trivially exist by virtue of the local cross section given by strip maps. Consider the submanifold $B^{\prime}$ of $B$ consisting of all fibers which contain a point of $\tau$, with the natural differentiable structure in which it is locally the product space of the fiber by the parameter along $\tau$. We define a vector field $W$ on $B^{\prime}$ by translating the tangent vector to $\tau$ up and down each fiber in $B^{\prime}$ under the action of $G$ on $B$. Then $H W$ is a vector field on $B^{\prime}$ any of whose integral curves will be such a $\sigma$. In terms of parallel translation we thus can consider the holonomy group of a connexion on $E(M)$ to be a group of linear transformations on the tangent space at a point of $M$.

A connexion $H$ on $B$ gives rise to a 1 -form $\omega$, the values of $\omega$ lying in the Lie algebra $L$ of the group $G$ of the bundle. $\omega$ is defined by: if $t \in B_{b}$ then $\omega(t)=$ the element of $L$ generated by $V t$, i.e. $\omega(t)$ is the unique $A \in L$ with $(q A)_{b}=V t$. Clearly $\omega$ has the following properties.

(1) $\omega$ is differentiable $\left({ }^{6}\right)$,

(2) if $t$ is vertical, then $\omega(t)$ is the element of $L$ generated by $t$,

(3) $\omega \circ R_{g}=\left(a d g^{-1}\right) \omega$, i.e. if $t \in B_{b}$ then $\omega\left(R_{g} t\right)=\left(a d g^{-1}\right)(\omega(t))$.

We have (3) because $V R_{g} t=R_{g} V t$ and $R_{g} A=\left(a d g^{-1}\right) A$ for $A \in L$. Conversely, any 1 -form $\omega$ on $B$ with values in $L$ and satisfying (1), (2), (3) gives rise to a connexion $H$ defined by: $H_{b}=\left[t \in B_{b} \mid \omega(t)=0\right]$. We call $\omega$ the 1-form of the connexion.

In differentiating a real-valued differentiable differential $p$-form $\theta$ we shall frequently make use of the formula:

(6) For a form $\omega$ whose values lie in a finite-dimensional linear space over $R$, differentiability means that for every linear functional $f$ from the vector space to $R, f \circ \omega$ is differentiable. 


$$
\begin{aligned}
& d \theta\left(W_{1}, \cdots, W_{p+1}\right) \\
& =\frac{1}{p+1} \sum_{i=1}^{p+1}(-1)^{i-1} W_{i}\left(\theta\left(W_{1}, \cdots, \widehat{W}_{i}, \cdots, W_{p+1}\right)\right. \\
& \quad+\frac{1}{p+1} \sum_{i<j}(-1)^{i+j} \theta\left(\left[W_{i}, W_{j}\right], W_{1}, \cdots, \widehat{W}_{i}, \cdots, \widehat{W}_{j}, \cdots, W_{p+1}\right)
\end{aligned}
$$

which can be used to define $d \theta$. I.e. this formula holds for any $p+1$ differentiable vector fields $W_{1}, \cdots, W_{p+1}$, and if this formula holds at a point for all choices of $W_{1}, \cdots, W_{p+1}$ from a family of vector fields which span the tangent space at that point, then this determines $d \theta$ at the point. If $\theta$ is a differentiable differential form with values in a finite-dimensional vector space over $R$, we define $d \theta$ in the usual way via linear functionals, i.e. $d \theta$ is the unique form such that $f \circ d \theta=d(f \circ \theta)$ for all such linear functionals $f$.

Definition. If $H$ is a connexion or a principle bundle with bundle space $B$ we define the covariant derivative $D \theta$ of a differentiable $p$-form $\theta$ on $B$ (when the values of $\theta$ lie in any finite-dimensional vector space over $R$ ) to be the $(p+1)$-form on $B$ defined by

$$
\text { (D9) }\left(t_{1}, \cdots, t_{p+1}\right)=d \theta\left(H t_{1}, \cdots, H t_{p+1}\right)
$$

where $t_{1}, \cdots, t_{p}$ are any elements of $B_{b}$. I.e. $D$ is the exterior derivative followed by the dual of the projection $H$. Clearly $D \theta$ is differentiable.

Definition. If $H$ is a connexion whose 1 -form is $\omega$, we define the curvature form $\Omega$ of the connexion by $\Omega=D \omega$.

It is easily seen that $\Omega \circ R_{g}=\left(a d g^{-1}\right) \Omega$, i.e. $\Omega\left(R_{g} t_{1}, R_{g} t_{2}\right)=a d g^{-1} \Omega\left(t_{1}, t_{2}\right)$ for any $t_{1}, t_{2} \in B_{b}$. This follows from the corresponding fact for $\omega$ and the facts (which are verified by simple computations) that if a form $\theta$ with values in $L$ satisfies this relation, then so do $d \theta$ and $H^{*} \theta$, where $H^{*}$ is the dual of $H$, i.e. $\left(H^{*} \theta\right)\left(t_{1}, \cdots, t_{p}\right)=\theta\left(H t_{1}, \cdots, H t_{p}\right)$.

We now explain, in the case of a bundle of bases $E(M)$, the geometric reason for calling this $\Omega$ the curvature. We show that $\Omega$ defines, at each $m \in M$ and for each $s, s^{\prime} \in M_{m}$, a linear transformation $T_{s, s^{\prime}}$, where this transformation can be considered as the effect of translating vectors parallel to themselves around the "infinitesimal parallelogram" spanned by $s$ and $s^{\prime}$; so this transformation, as a function of $s$ and $s^{\prime}$, is a measure of how $M$ "curves" at $m$-this "curving" depending on the connexion. We also point out in the case of a Riemannian connexion how to get the Riemannian curvature, which is a number depending on $m, s, s^{\prime}$, from $T_{8,8^{\prime}}$.

Let $s, s^{\prime} \in M$, consider any $b$ in $E(M)$ lying over $m$ (i.e. $\pi b=m$ ) and any horizontal differentiable vector fields $K$ and $K^{\prime}$ defined on a neighborhood of $b$ such that $\pi K(b)=s, \pi K^{\prime}(b)=s^{\prime}$. We define for each small positive number $\delta$ a "circuit" $\gamma_{\delta}$, which is a piecewise differentiable horizontal curve consisting of four differentiable pieces, as follows. Take the integral curve $\rho$ of $K$ with 
$\rho(0)=b$, then an integral curve $\rho^{\prime}$ of $K^{\prime}$ with $\rho^{\prime}(0)=\rho(\delta)$, then an integral curve $\sigma$ of $K$ with $\sigma(0)=\rho^{\prime}(\delta)$, then an integral curve $\sigma^{\prime}$ of $K^{\prime}$ with $\sigma^{\prime}(0)$ $=\sigma(-\delta)$. The circuit $\gamma_{\delta}$ will be the curve obtained by going along $\rho$ from $\rho(0)$ to $\rho(\delta)$, then along $\rho^{\prime}$ from $\rho(\delta)=\rho^{\prime}(0)$ to $\rho^{\prime}(\delta)$, then along $\sigma$ from $\rho^{\prime}(\delta)$ $=\sigma(0)$ to $\sigma(-\delta)$, then along $\sigma^{\prime}$ from $\sigma(-\delta)=\sigma^{\prime}(0)$ to $\sigma^{\prime}(-\delta)$. Next we consider the curve made up of the end points of these circuits, suitably parametrized, i.e. we define $\gamma(t)=$ final point of $\gamma_{t^{1 / 2}}$. (This is our definition of $\gamma$ for $t>0$; for $t<0$ we can define it similarly or we can ignore all $t<0$.) If we define $\gamma(0)=b$, then $\gamma$ is a differentiable curve whose tangent vector at $b$ is $\left[K, K^{\prime}\right](b)$ (this is just the geometrical significance of the bracket operation, applied to our $K$ and $K^{\prime}$ ). Let $\eta_{\delta}$ and $\eta$ be the projection of $\gamma_{\delta}$ and $\gamma$ into $M$. Note that $\eta_{\delta}$ is a closed curve if and only if the final point of $\gamma_{\delta}$ lies on the same fiber as $b$ and in this case $b=\left(m, e_{1}, \cdots, e_{d}\right)$, the final point of $\gamma_{\delta}$ is $\left(m, f_{1}, \cdots, f_{d}\right)$, and parallel translation around $\eta_{\delta}$ is the linear transformation taking $e_{i} \rightarrow f_{i}$; this parallel translation gives rise to an element $g \in G$ ( $G$ the linear group), namely the $g$ such that $R_{g}\left(m, e_{1}, \cdots, e_{d}\right)$ $=\left(n, f_{1}, \cdots, f_{d}\right)$. In general $\eta_{\delta}$ is not closed and the final point of $\gamma_{\delta}$ does not lie on the same fiber as $b$, however the vertical component of the tangent vector to $\gamma$ at $b$ is an infinitesimal analogue of the transformation mentioned above carrying $e_{i} \rightarrow f_{i}$. The element $A$ of $L$ ( $L$ the Lie algebra of $G$ ) generated by the vertical component of the tangent vector to $\gamma$ at $b$ is an infinitesimal analogue of the above $g$ and we point out in the next paragraph how, through $A$, the vertical component of this tangent gives rise to a transformation $T_{s, s^{\prime}}$ on $M_{m}$. This $T_{s, s^{\prime}}$, as a function of $s, s^{\prime}, m\left(s\right.$ and $s^{\prime}$ in $\left.M_{m}\right)$, determines the curvature form $\Omega$ and conversely.

By definition of the 1 -form $\omega$ of the connexion we have $A=\omega\left(\left[K, K^{\prime}\right](b)\right)$ and a simple calculation (which is given in the proof of Theorem 2 below) shows $\omega\left(\left[K, K^{\prime}\right](b)\right)=-\Omega\left(K(b), K^{\prime}(b)\right)$. Thus we have shown that if $t$ and $t^{\prime}$ are the unique horizontal elements of $B_{b}$ which project to $s$ and $s^{\prime}$ (we can discard the vector fields $K$ and $K^{\prime}$ at this point), then the element $A$ of $L$ generated by the vertical component of the tangent vector to $\gamma$ at $b$ is given by $A=-\Omega\left(t, t^{\prime}\right)$. Because $G$ is the linear group, each $A \in L$ gives rise to a linear transformation $T_{A}$ on $R^{d}$ defined by: $f\left(T_{A} x\right)=A(e) f_{x}$, where $f$ is any linear function on $R^{d}$ and $f_{x}$ is the function defined on $G$ by $f_{x}(g)=f(g x)$, for $x \in R^{d}$. (In terms of the usual coordinates $x_{i j}$ on $G$ this just defines $T_{A}$ as the linear transformation whose matrix with respect to the base $\delta_{1}, \cdots, \delta_{d}$ is $\left(A(e) x_{i j}\right)$.) We define $T_{s, s^{\prime}}=I_{b}^{-1} \circ T_{A} \circ I_{b}$, where $I_{b}$ is the mapping defined above of $M_{m}$ onto $R^{d}$, associated with $b$. It remains only to show this definition is independent of the point $b$ over $m-$ a consequence of the behavior of $\Omega$ under $R_{g}$. To prove this let $b g$ be another point over $m, t_{1}$ and $t_{1}^{\prime}$ the horizontal vectors at $b g$ that project to $s$ and $s^{\prime}$, so $R_{g} t=t_{1}, R_{g} t^{\prime}=t_{1}^{\prime}$, and let $A_{1}=-\Omega\left(t_{1}, t_{1}^{\prime}\right)$. Because $\Omega\left(t_{1}, t_{1}^{\prime}\right)=\Omega\left(R_{g} t, R_{g} t^{\prime}\right)=a d g^{-1} \Omega\left(t, t^{\prime}\right)$ we have 
$T_{A}=g^{-1} T_{A} g$. Then using that $I_{b q}=g^{-1} \circ I_{b}$ we have $I_{b q}^{-1} \circ T_{A} \circ I_{b g}=I_{b}^{-1}$ $\circ g \circ g^{-1} \circ T_{A} \circ g \circ g^{-1} \circ I_{b}=I_{b}^{-1} \circ T_{A} \circ I_{b}$.

Consider now a Riemannian connexion on $E(M)$ (the Riemannian connexion is usually considered on the bundle of frames $F(M)$ but may equally well be considered on $E(M)$ - or any principal bundle containing $F(M)$ as a sub-bundle). I.e. we have a scalar product $\langle$,$\rangle on each M_{m}$ which is a differentiable function of $m$ and $H$ is the unique connexion on $E(M)$ with torsion zero (torsion is discussed at the end of this section) and such that parallel translation preserves the scalar product. Then the function considered above which assigns to each $s$ and $s^{\prime}$ in $M_{m}$ the linear transformation $T_{s, 8^{\prime}}$ can be characterized by the simpler function $K$ which assigns to each such $s$ and $s^{\prime}$ the number $K=\left\langle T_{s, s^{\prime}} s, s^{\prime}\right\rangle / \alpha\left(s, s^{\prime}\right)^{2}$ where $\alpha\left(s, s^{\prime}\right)$ is the "area" of the parallelogram spanned by $s$ and $s^{\prime}$, i.e. $\alpha\left(s, s^{\prime}\right)=\left(\|s\|\left\|^{2}\right\| s^{\prime} \|^{2}-\left\langle s, s^{\prime}\right\rangle^{2}\right)^{1 / 2}$. It is easily seen that $K\left(s, s^{\prime}\right)$ does not depend on $s$ and $s^{\prime}$ but only on the plane section (i.e. linear subspace of $M_{m}$ ) spanned by $s$ and $s^{\prime}$. This $K$ is the Riemannian curvature.

For the proof of the structural equation of a connexion we need the following lemma, which might be called the vector field formulation of the structural equation.

Lemma. Let $H$ be any connexion on a principal bundle with bundle space $B$, and 2 as above. If $K$ is any horizontal vector field on $B$ and $Q \in 2$ then $[K, Q]$ is horizontal.

Proof. We need first the fact: if $A$ and $B$ are vector fields on a Lie group $G$ with $A$ left invariant and $B$ right invariant, then $[A, B]=0$. This can be proved as follows. Define vector fields $A^{\prime}$ and $B^{\prime}$ on $G \times G$ by: $A^{\prime}(g, h)$ $=J_{\bullet} A(h), B^{\prime}(g, h)=I_{h} B(g)$ where

$$
I_{h}: G \rightarrow G \times G: g \rightarrow(g, h) ; \quad J_{g}: G \rightarrow G \times G ; \quad h \rightarrow(g, h) .
$$

Then $\left[A^{\prime}, B^{\prime}\right]=0$ for if we consider any $(g, h) \in G \times G$ and choose coordinates $x_{1}, \cdots, x_{n}$ on a neighborhood of $g$, coordinates $y_{1}, \cdots, y_{n}$ on a neighborhood of $h$, then $A^{\prime}=\sum a_{i} Y^{i}, B^{\prime}=\sum b_{i} X^{i}$ where the $a_{i}$ depend only on the $y_{j}$-coordinates and the $b_{i}$ depend only on the $x_{j}$-coordinates. Computation shows $\Phi A^{\prime}=A$ and $\Phi B^{\prime}=B$ where $\Phi$ denotes group multiplication in $G$. Hence $[A, B]=0$.

It follows that for any vertical vector field $W$ on $\pi^{-1}(0)$ ( 0 any open subset of $M$ ) which is invariant under the action of $G$ on $B$ we have $[W, Q]=0$ for all $Q \in 2$.

Now consider any strip map $\phi: 0 \times G \rightarrow B$ where there exists a coordinate system $x_{1}, \cdots, x_{d}$ of $M$ with domain $O$ and let $x_{j}=x_{j} \circ \pi$. We call a coordinate system of $B$ special if it consists of the functions $y_{1}, \cdots, y_{d}$ plus some functions $z_{1}, \cdots, z_{k}$ which are carried over by $\phi$ from a coordinate 
system $v_{1}, \cdots, v_{k}$ of $G$ (i.e. $z_{j}=v_{j} \circ p \circ \phi^{-1}$ where $\left.p: 0 \times G \rightarrow G:(m, g) \rightarrow g\right)$ ); if the domain of $v_{1}, \cdots, v_{k}$ is $P$, then the domain of this special system is $\phi(0 \times P)$. If we have two special coordinate systems (special, that is, for the same $\phi$ and $\left.x_{1}, \cdots, x_{d}\right)$ then at any point $b$ common to their domains the partial derivative with respect to $y_{j}$ at $b$ defined through the two coordinate systems is easily seen to be the same tangent vector and we denote the vector field thus defined throughout $\pi^{-1}(0)$ by $Y^{i}$, i.e. $Y^{i}(b)$ is the partial derivative with respect to $y_{j}$, where $y_{1}, \cdots, y_{d}, z_{1}, \cdots, z_{k}$ is any special coordinate system whose domain includes $b$. Then $\pi Y^{i}=X^{j}$ and $R_{g} Y^{i}=Y^{j}$. Write $Y^{i}=K^{j}+W^{i}$ with $K^{j}$ horizontal and $W^{j}$ vertical. We next show $\left[K^{i}, Q\right]=0$ for all $Q \in 2$, by showing separately that $\left[Y^{k}, Q\right]=0$ and $\left[W^{i}, Q\right]$ $=0$.

To prove $\left[Y^{j}, Q\right]=0$ consider any $b \in \pi^{-1}(0)$ and a special coordinate system $y_{1}, \cdots, y_{d}, z_{1}, \cdots, z_{k}$ at $b$. The expression for $Q$ in these coordinates involves only the $z_{i}$ and with coefficients that depend only on the $z_{i}$. Hence $\left[Y^{i}, Q\right]=0$. To prove $\left[K^{j}, Q\right]=0$ it is sufficient, by a remark made above, to show $R_{g} K^{i}=K^{j}$. Because $R_{g} K^{i}$ is horizontal, $R_{g} W^{j}$ is vertical, and the horizontal vectors at any point are a linear complement to the vertical vectors, this follows from

$$
K^{j}+W^{i}=Y^{j}=R_{g} Y^{j}=R_{\theta} K^{j}+R_{g} W^{j} .
$$

Now consider any horizontal vector field $K$ defined on a neighborhood of any $b \in \phi(0 \times G)$. Using the above notation, the $K^{j}$ form a base for the horizontal vectors throughout $\phi(0 \times G)$ (because of the iso of $H_{b}$ with $M_{m}$ and the fact that the $X^{i}$ are a base for $M_{m}$ ) so $K=\sum a_{j} K^{i}$. Then

$$
[K, Q]=\left[\sum a_{j} K^{j}, Q\right]=\sum a_{j}\left[K^{i}, Q\right]-\sum\left(Q a_{j}\right) K^{i}=-\sum\left(Q a_{j}\right) K^{i} .
$$

THEOREM 1. (The structural equation of a connexion). If $H$ is any connexion on a principal bundle with bundle space $B, \omega$ is the 1-form of the connexion, and $\Omega$ is the curvature form of the connexion, then

$$
d \omega=-[\omega, \omega] / 2+\Omega,
$$

i.e. for any $b \in B$ and $s, t \in B_{b}$ we have

$$
d \omega(s, t)=-[\omega(s), \omega(t)] / 2+\Omega(s, t) .
$$

Proof. The formula mentioned above for the differential of a real-valued differentiable differential $p$-form $\theta$ becomes, in case $p=1$,

$$
d \theta\left(W, W^{\prime}\right)=\left(W \theta\left(W^{\prime}\right)-W^{\prime} \theta(W)-\theta\left(\left[W, W^{\prime}\right]\right)\right) / 2,
$$

where $W$ and $W^{\prime}$ are any differentiable vector fields. Further, if $\eta$ is a 2-form and we wish to show $d \theta=\eta$, it is sufficient to find for each $b \in B$ a family of vector fields $W^{\prime}, \cdots, W^{r}$ for which the $W^{i}(b)$ span $B_{b}$ and such that $d \theta\left(W^{i}(b), W^{i}(b)\right)=\eta\left(W^{i}(b), W^{j}(b)\right)$ for all $i, j$. 
The differential forms of our theorem have values in a Lie algebra so we must prove, for any linear functional $f$ on that Lie algebra, that

$$
f\left(d \omega\left(W, W^{\prime}\right)\right)=-f\left(\left[\omega(W), \omega\left(W^{\prime}\right)\right]\right) / 2+f\left(\Omega\left(W, W^{\prime}\right)\right) .
$$

Letting $\theta=f \circ \omega$ this becomes

$$
d \theta\left(W, W^{\prime}\right)=-f\left(\left[\omega(W), \omega\left(W^{\prime}\right)\right] / 2+d \theta\left(H W, H W^{\prime}\right)\right.
$$

and using the above formula for $d \theta$ this becomes

$$
\begin{array}{r}
W \theta\left(W^{\prime}\right)-W^{\prime} \theta(W)-\theta\left(\left[W, W^{\prime}\right]\right)=-f\left(\left[\omega(W), \omega\left(W^{\prime}\right)\right)+(H W)\left(\theta\left(H W^{\prime}\right)\right)\right. \\
-\left(H W^{\prime}\right)(\theta(H W))-\theta\left(\left[H W, H W^{\prime}\right]\right) .
\end{array}
$$

It is sufficient to prove this at each $b \in B$, for all choices of $W$ and $W^{\prime}$ from any family $W^{\prime}, \cdots, W^{r}$ which are defined on a neighborhood of $b$ and such that $W^{i}(b)$ span $B_{b}$. We choose such a family consisting of any $Q^{\prime}, \cdots, Q^{k}$ which span 2 plus any horizontal $K^{\prime}, \cdots, K^{d}$ for which the $K^{i}(b)$ span $H_{b}$.

The desired formula holds whenever $W$ is a $K^{i}$ and $W^{\prime}$ a $K^{j}$, for then $\omega(W)=0, \omega\left(W^{\prime}\right)=0$, hence $f\left(\left[\omega(W), \omega\left(W^{\prime}\right)\right]\right)=0$; and $W=H W, W^{\prime}=H W^{\prime}$, so the two sides are identical. If $W$ is a $Q^{i}$ and $W^{\prime}$ is a $K^{j}$, then $\omega\left(W^{\prime}\right)=0$ hence $f\left(\left[(\omega W), \omega\left(W^{\prime}\right)\right]\right)=0$, and $H W=0$ so the entire right side is zero. In this case the left side is also zero, $\theta\left(\left[W, W^{\prime}\right]\right)$ being zero because the previous lemma shows in this case that $\left[W, W^{\prime}\right]$ is horizontal, $\theta\left(W^{\prime}\right)$ being zero because $W^{\prime}$ is horizontal, and $W^{\prime} \theta(W)$ being zero because $\theta\left(W^{\prime}\right)$ is a constant function. If $W$ is a $Q^{i}$ and $W^{\prime}$ is a $Q^{j}$, then (because $q$ is a Lie algebra iso) $f\left(\left[\omega(W), \omega\left(W^{\prime}\right)\right]\right)=f\left(\omega\left[W, W^{\prime}\right]\right)=\theta\left(\left[W, W^{\prime}\right]\right)$ and $H W$ and $H W^{\prime}$ are 0 so the left side reduces to $-\theta\left(\left[W, W^{\prime}\right]\right)$. Because both $\theta(W)$ and $\theta\left(W^{\prime}\right)$ are constant functions the right side also reduces to $-\theta\left(\left[W, W^{\prime}\right]\right)$. Hence the formula is proved.

In the special case of a connexion on a bundle of bases, and if the elements of the Lie algebra of the linear group are represented in the usual way by matrices, then $\omega(t)$ is represented by $\left(\omega_{i j}(t)\right), \Omega(s, t)$ is represented by $\left(\Omega_{i j}(s, t)\right)$, and the above formula becomes

$$
d \omega_{i j}=-\sum \omega_{i \alpha} \wedge \omega_{\alpha j}+\Omega_{i j}
$$

In this case, however, one gets the further formula

$$
d \omega_{i}=-\sum \omega_{\alpha i} \wedge \omega_{\alpha}+\Omega_{i}
$$

which we now explain. One defines 1-forms $\omega_{1}, \cdots, \omega_{d}$ on $E(M)$ by: if $t \in E(M)_{b}$ and $b=\left(m, e_{1}, \cdots, e_{d}\right)$, then $\omega_{i}(t)$ is the $i$ th coefficient of $\pi t$ when $\pi t$ is expressed linearly in terms of the base $e_{1}, \cdots, e_{d}$ of $M_{m}$. Then $\Omega_{i}$ is defined to be the covariant derivative of $\omega_{i}$ and this formula is proved similarly to the other; the $\Omega_{i}$ are called the torsion forms of the connexion. Let $E^{1}, \cdots, E^{d}$ be the vector fields defined on $E(M)$ by: if $b=\left(m, e_{1}, \cdots, e_{d}\right)$, then $E^{i}(b)$ is the unique element of $H_{b}$ that projects to $e_{i}$ under $\pi$. One verifies 
easily that torsion zero (i.e. all $\Omega_{i}=0$ ) is equivalent to the statement that $\left[E^{i}, E^{j}\right]$ is vertical for all $i, j$. In fact the role of the $\Omega_{i}$ is precisely to describe the horizontal components of the $\left[E^{k}, E^{r}\right]$, just as the role of the $\Omega_{i j}$ is to describe their vertical components; the significant facts about such a connexion may be described either through the $\left[E^{i}, E^{j}\right]$ or through the $\Omega_{i j}$ and $\Omega_{k}$. A more elegant way of interpreting the above formula for $d \omega_{i}$, however, is to pass from the bundle $E(M)$ to a larger bundle whose group is the affine group. The connexion $H$ on $E(M)$ gives rise naturally to a connexion $K$ on the larger bundle, and with the following properties. Let $\Theta$ be the curvature form of $K$. Then $\Theta$ decomposes into $\Theta^{\prime}+\Theta^{\prime \prime}$ through the decomposition of the Lie algebra of the affine group into the subalgebras corresponding to the linear transformations and the translations. $\Theta^{\prime}$ is essentially the curvature form of $H$ and $\Theta^{\prime \prime}$ is essentially the torsion. The structural equation for $K$ can be similarly decomposed into two parts, the two parts being essentially the equations for $d \omega_{i j}$ and for $d \omega_{i}$.

2. Curvature as generator of the holonomy group. In this section we consider a connexion $H$ on a principal bundle $(B, M, G, \pi, \Phi)$. We assume further that $M$ is connected and that its fundamental group has at most a countable number of elements. We denote the 1 -form of the connexion by $\omega$ and the curvature form by $\Omega$. The Lie algebra of $G$ will be denoted by $L$. If $b \in B$, then the holonomy group and null holonomy group associated with $b$ will be denoted by $G(b)$ and $G_{0}(b)$, while the arc-component of the identity of $G(b)$ will be denoted by $G(b)^{0}$. B $B(b)$ will denote the subset of $B$ consisting of all points which can be joined to $b$ by piece-wise differentiable horizontal curves. This notation will be kept fixed throughout the section.

Theorem 2. For any $b \in B$ let $L(b)$ be the subalgebra of $L$ generated by all $\Omega(s, t)$ where $s$ and $t$ run through all pairs of tangent vectors to $B$ at all points of $B(b)$. Then the subgroup of $G$ generated by $L(b)$ is $G_{0}(b)$.

We prove this via a sequence of lemmas. The first two are needed to prove $B(b)$ a submanifold of $B$, which fact is important in the proof of this theorem.

LemMa 1. Let $\phi$ be a strip map of the bundle, $\phi=O \times G \rightarrow B$, where $O$ is an open submanifold of $M$. Suppose that $O$ is the domain of a coordinate system $x_{1}, \cdots, x_{d}$ of $M$ with center $m_{0}$ (i.e. all $x_{i}\left(m_{0}\right)=O$ ) and that $O$ is an open ball with respect to these coordinates. Let $b_{0}=\phi\left(m_{0}, e\right)$. We now define a mapping $\alpha$ of $O \rightarrow G$ ( $\alpha$ will depend on the coordinates $x_{i}$ ) and the assertion of this lemma is that $\alpha$ is differentiable on some neighborhood $P$ of $m_{0}$. To define $\alpha$ consider, for each $m \in 0$, the ray $\rho_{m}$ (i.e. $\rho_{m}$ is a ray with respect to the coordinates $x_{i}$ ) from $m_{0}$ to $m$, parametrized so that $\rho_{m}(0)=m_{0}, \rho_{m}(1)=m$. Lying above $\rho_{m}$ we have curves $\sigma_{m}$ and $\tau_{m}$ in $B$ defined as follows. $\sigma_{m}$ is the unique horizontal curve in $B$ starting at $b_{0}$ and lying over $\rho_{m} . \tau_{m}$ is defined by: $\tau_{m}(u)=\phi\left(\rho_{m}(u), e\right)$. Then $\sigma_{m}(1)$ $=\tau_{m}(1) g$ for a unique $g \in G$ and we define $\alpha(m)=g$. 
Proof. Let $v_{1}, \cdots, v_{k}$ be any coordinate system on some neighborhood $U$ of $e$ in $G$. To prove the lemma we shall show the functions $v_{j} \circ \alpha$ are differentiable on some neighborhood $P$ of $m_{0}$. The $x_{i}$ and $v_{j}$ give coordinates in the usual way on $\phi(O \times G)$ which are carried by $\phi$ to coordinates on $B$; we denote these coordinates, whose domain is $\phi(O \times U)$, by $y_{1}, \cdots, y_{k+d}$.

We first prove the existence of $\sigma_{m}$ as stated in the lemma. We define $\sigma_{m}$ to be the integral curve with $\sigma_{m}(0)=b_{0}$ of the vector field $H\left(\sum_{i=1}^{d} x_{i}(m) Y^{i}\right)-$ this vector field is defined on $\pi^{-1}(0)$ and is differentiable by condition (3) in the definition of a connexion, hence $\sigma_{m}$ is a horizontal differentiable curve. We have $\pi \circ \sigma_{m}=\rho_{m}$ because $\pi H\left(\sum_{i=1}^{d} x_{i}(m) Y^{i}\right)=\sum x_{i}(m) X^{i}$, which implies $\pi \circ \sigma_{m}$ is an integral curve of $\sum x_{i}(m) X^{i}$, as is also $\rho_{m}$, hence $\pi \circ \sigma_{m}=\rho_{m}$. Uniqueness of $\sigma_{m}$ follows from the uniqueness of integral curves, for any such $\sigma_{m}$ is an integral curve of $H\left(\sum_{i=1}^{d} x_{i}(m) Y^{i}\right)$.

If $m \in O$ we shall write $u m$ for $\rho_{m}(u)$. Since $\phi(m, e) g=\phi(m, g)$ we have $\phi\left(m, \alpha(m)=\phi(m, e) \alpha(m)=\sigma_{m}(1)\right.$, and since $\sigma_{m}(u)=\sigma_{u m}(1)$ we then have $\phi(u m, \alpha(u m))=\sigma_{m}(u)$. Because $\sigma_{m}$ is a differentiable curve in $B$ we have, for each $m \in O$, the existence of $u_{m}>0$ such that $\sigma_{m}(u) \in U$ for $u<u_{m}$, and we take $u_{m}$ to be the largest possible such number. Then $v_{j}(\alpha(u m))$ $=y_{j+d}(\phi(u m, \alpha(u m)))=y_{j+d}\left(\sigma_{m}(u)\right)$ for $u<u_{m}$.

Because $\sigma_{m}$ is an integral curve of $H\left(\sum_{i=1}^{d} x_{i}(m) Y^{i}\right)$ we have

$$
\left(D\left(y_{j+d} \circ \sigma_{m}\right)\right)(u)=\left(d y_{j+d}\left(H\left(\sum_{i=1}^{d} y_{i} Y^{i}\right)\right)\right)\left(\sigma_{m}(u)\right)
$$

where $D$ denotes the usual differentiation operator on $R$. From the definition of a connexion it follows that the function $d y_{j+d}\left(H\left(\sum_{i=1}^{d} y_{i} Y^{i}\right)\right)$ is a differentiable function and hence can be expressed on a neighborhood of $b_{0}$ as a differentiable function of the $y_{r}$. This shows the functions $y_{r} \circ \sigma_{m}$ are solutions of a system of ordinary differential equations with differentiable functions on the "right side." It follows that the functions $f_{j}$ defined by: $f_{j}(u, m)$ $=\left(y_{j} \circ \sigma_{m}\right)(u)$ for $u<u_{m}$ are defined and differentiable on some neighborhood of $\left(O, m_{0}\right)$.

Because $\sigma_{m}(u)=\sigma_{u m}(1)$ it follows that $\sigma_{m}(1) \in U$ for $m$ in some neighborhood $P$ of $m_{0}$ and then, because $\left(v_{j} \circ \alpha\right)(m)=y_{j}\left(\sigma_{m}(1)\right)=f_{j}(1, m)$, we have that $v_{j} \circ \alpha$ is differentiable on $P$.

LeMmA 2. Let $m_{0} \in M, b_{0}$ be any point of $B$ lying over $m_{0}$, and $x_{1}, \cdots, x_{d}$ any coordinate system of $M$ whose domain includes $m_{0}$ and has center at $m_{0}$. Then there exists a strip map $\psi: P \times G \rightarrow B$, where $P$ is an open ball with respect to the coordinates $x_{i}$, such that $\psi\left(m_{0}, e\right)=b$ and for every ray $\rho_{m}$ from $m_{0}$ to $m$ in $P$ (with respect to these coordinates) the curve $\psi\left(\rho_{m}, e\right)$ in $B$ is horizontal. We call such a strip map canonical with respect to the coordinates $x_{i}$.

Proof. Let $\phi$ be any strip map taking $O \times G \rightarrow B$, with $\phi\left(m_{0}, e\right)=b_{0}$, where $O$ is an open subset of the domain of the coordinates $x_{i}$. We define $\alpha$, depending 
on $\phi$ and the $x_{i}$, as in the previous lemma. Let $P$ be any neighborhood of $m_{0}$ on which $\alpha$ is differentiable and such that $P$ is a ball with respect to the coordinates $x_{i}$. We define $\psi$ by: $\psi(m, g)=\phi(m, \alpha(m) g) . \psi$ is differentiable because $\alpha$ and $\phi$ are differentiable. $\psi$ is a strip map because, for any strip map $\chi$, $\left(\chi_{m}^{-1} \circ \psi_{m}\right)(g)=\left(\chi_{m}^{-1} \circ \phi_{m}\right)(\alpha(m) g)$, so if $\chi_{m}^{-1} \circ \phi_{m}=L_{h}\left({ }^{7}\right)$ then $\chi_{m}^{-1} \circ \psi_{m}=L_{h} \alpha_{(m)}$. And $\psi\left(\rho_{m}, e\right)$ is horizontal because $\psi\left(\rho_{m}(u), e\right)=\phi\left(\rho_{m}(u), \quad \alpha\left(\rho_{m}(u)\right)\right.$ $=R \alpha\left(\rho_{m}(u)\right) \phi\left(\rho_{m}(u), e\right)$.

We now define the differentiable structure in which $B(b)$ is a submanifold of $B$. First note that $G(b)^{0}$, being an arcwise connected subgroup of the Lie group $G$, is a differentiable subgroup of $G\left(^{8}\right)$ [8]. Hence there exists a coordinate system $v_{1}, \cdots, v_{k}$ of $G$ whose domain includes $e$ and which is adapted to $G(b)^{0}$, i.e. the slices $\left[g \mid v_{i}(g)=a_{i}\right.$ for all $\left.i=j+1, \cdots, k\right]$ are contained in left cosets of $G(b)^{0}$ and the slice $S^{0}$ with all these $a_{i}=0$ is contained in $G(b)^{0}$. Now consider any $c \in B(b)$ and we shall define some coordinate systems on $B(b)$ at $c$. Let $x_{1}, \cdots, x_{d}$ be any coordinate system of $M$ whose domain contains $m^{\prime}=\pi c$ and $\psi: P \times G \rightarrow B$ any canonical strip map with respect to these taking $\left(m^{\prime}, e\right) \rightarrow c$. Then $\psi\left(P \times S_{0}\right) \subseteq B(b)$. The coordinates $x_{1}, \cdots, x_{d}$ on $P$ and $v_{1}, \cdots, v_{j}$ (the $v_{i}$ and $j$ as above) induce coordinates in the usual way on $P \times S_{0}$ which are carried over to coordinates on $\psi\left(P \times S_{0}\right)$ by $\psi$. We should not yet refer to these functions on $\psi\left(P \times S_{0}\right)$ as coordinates but it can be shown in routine fashion that such systems of functions on $B(b)$ (depending on choices of $\left.x_{1}, \cdots, x_{d}, v_{1}, \cdots, v_{k}, c, \psi\right)$ satisfy the usual compatibility conditions and cover $B(b)$ so they give rise to a unique differentiable structure in which they are coordinate systems. Obviously $B(b)$ is a submanifold of $B$.

\section{Lemma 3. If $Q \in 2, c \in B(b), d \in B(b), Q(c) \in B(b) c$, then $Q_{d} \in B(b)_{d}$.}

Proof. We begin with two special cases. The first is where $c$ and $d$ lie on the same fiber, so $d=c g$ and because $c$ and $d$ are in $B(b)$ we have $g \in G(b)$. Consider any strip map $\phi: O \times G \rightarrow B$ such that $\phi(m, e)=c$. It is trivial that $\phi \circ L_{g} \circ \phi^{-1}$ maps $B(b) \cap \pi^{-1}(0)$ diffeomorphically onto itself and takes $c \rightarrow d$, $Q(c) \rightarrow Q(d)$. Hence $Q(d) \in B(b)_{d}$ in this case. The second special case is where there exists a coordinate system $x_{1}, \cdots, x_{d}$ of $M$ with center at $m=\pi c$ and a canonical (with respect to these $x_{i}$ ) strip map $\psi: P \times G \rightarrow B$, such that $d$ $=\psi\left(m^{\prime}, e\right)$ for some $m^{\prime} \in P$. Let $v_{1}, \cdots, v_{k}$ be coordinates of $G$ on a neighborhood of $e$, adapted to $G(b)^{0}$, so we get coordinates $y_{1}, \cdots, y_{d}, y_{d+1}, \cdots, y_{d+j}$ (these are the coordinates obtained from $x_{1}, \cdots, x_{d}$ and $v_{1}, \cdots, v_{k}$ as in the definition of the differentiable structure on $B(b))$ of $B(b)$ whose domain is a $B(b)$-neighborhood of $c$. Because $Q \in 2$ and $Q(c) \in B(b)_{c}$ we have $Q(c)$ $=\sum_{i=d+1}^{d+j} a_{i} Y^{i}(c)$, hence $Q(d)=\sum_{i=d+1}^{d+j} a_{i} Y^{i}(d)$, showing $Q(d) \in B(b)_{d}$. The

(7) $L_{h}$ denotes left multiplication by $h$ on $G$.

(8) That $G(b)^{0}$ is a Lie group was pointed out to us by Chevalley, who also found Yamabe's theorem independently. 
lemma now follows in general for any $c, d \in B(b)$ by a sequence of steps involving these special cases.

LEMMA 4. $G_{0}(b)=G(b)^{0}$.

Proof. $\left({ }^{9}\right)$ Clearly $G_{0}(b) \subseteq G(b)^{0}$ and since both are arcwise connected both are differentiable subgroups of $G$; hence $G_{0}(b)$ is a differentiable subgroup of $G(b)^{0}$. Now we show the existence of a mapping of $\pi_{1}(M)$ onto the left cosets of $G_{0}(b)$ in $G(b)^{0}$. Since $\pi_{1}(M)$ contains only countably many elements this will prove the left cosets of $G_{0}(b)$ in $G(b)^{0}$ are at most countable in number. This will imply $G_{0}(b)$ is open in $G(b)^{0}$ and hence, by connectedness, that $G_{0}(b)$ $=G(b)^{0}$.

For each $\alpha \in \pi_{1}(M)$ let $E(\alpha)=\left[g \in G(b)^{0} \mid\right.$ there exists a piecewise differentiable horizontal curve from $b$ to $b g$ whose projection into $M$ is in the homotopy class $\alpha$ ]. Each $E(\alpha)$ is contained in a left coset of $G_{0}(b)$, for if $g_{1}$ and $g_{2}$ are in $E(\alpha)$ let $\rho_{i}$ be a piecewise differentiable horizontal curve from $b$ to $b g_{i}$; then $\left(\rho^{-1} \circ R_{g_{2}{ }^{-1} g_{1}}\right) \cdot \rho_{1}$ is a piecewise differentiable horizontal curve from $b$ to $b g_{2}^{-1} g_{1}$ and its projection into $M$ is $\pi\left(\rho_{2}^{-1} \cdot \rho_{1}\right)$, showing $g_{2}^{-1} g_{1} \in G_{0}(b)$. Since each $g \in G(b)^{0}$ is in some $E(\alpha)$ this furnishes the desired mapping of $\pi_{1}(M)$ onto left cosets.

If $H$ is a connected differentiable subgroup of a Lie group $G$ with only countably many left cosets, then $H$ is open in $G$ because otherwise $H$ would be of measure 0 ( $H$ is trivially a measurable subset of $G$ with respect to the Haar measure of $G$ because, choosing coordinates adapted to $H$ on a neighborhood of $e$ in $G$, we see some $H$-neighborhood of $e$ is measurable, and then $H$ is a countable union of translates of this neighborhood) so $G$ itself would have measure 0 .

Proof of Theorem 2. We first describe, for each $c \in B(b)_{c}$, a subspace $\Delta_{c}$ of $B_{c}$, then prove $\Delta_{c}=B(b)_{c}$, from which the theorem will follow. Note that, trivially, $H_{c} \subseteq B(b)_{c}$ for $c \in B(b)$.

Consider any horizontal vector fields $K$ and $K^{\prime}$ defined on any open submanifold $O$ of $B$ and for each $d \in O \cap B(b)$ consider the vector field $Q \in \mathcal{Q}$ generated by $V\left(\left[K, K^{\prime}\right](d)\right)$ - so $Q$ is defined on all of $B$ and depends on $K, K^{\prime}$ and $d$. We do this for all such $K, K^{\prime}, d$, getting a subset $R$ of 2 , and define $\mathcal{L}(b)$ to be the Lie algebra of vector fields on $B$ generated by $R$. Clearly $\mathcal{L}(b) \subseteq 2$, and we shall prove later that $\mathcal{L}(b)=q(L(b))$. We define $\Delta_{c}$, for $c \in B(b)$, to be the subspace of $B_{c}$ spanned by $H_{c}$ and all $Q_{c}$ for which $Q \in \mathcal{L}(b)$.

Now we prove $\Delta_{c} \subseteq B(b)_{c}$ for all $c \in B(b)$. Clearly if $K$ is any horizontal vector field on an open subset of $B$, then $K(c) \in B(b)_{c}$. Hence if $K_{1}$ and $K_{2}$ are two such and $K_{1}^{\prime}, K_{2}^{\prime}$ are their restrictions to $B(b)$, then $\left[K_{1}^{\prime}, K_{2}^{\prime}\right](c)$ $=\left[K_{1}, K_{2}\right](c)$, so $B(b)_{c}$ contains $\left[K_{1}, K_{2}\right](c)$, and since it contains all horizontal vectors at $c, B(b)_{c}$ contains $V\left(\left[K_{1}, K_{2}\right](c)\right)$. This with Lemma 3 shows

(9) This lemma is an extension of one due to Borel and Lichnerowicz [1], and the proof is a variation on theirs. 
$B(b)_{c}$ contains also $Q(c)$ for any $Q \in \mathcal{Q}$ and $d \in B(b)$ with $V\left(\left[K_{1}, K_{2}\right](d)\right)$ $=Q(d)$. Finally, if $Q_{1}, Q_{2} \in 2$ and $Q_{1}(c), Q_{2}(c) \in B(b)_{c}$ then $\left[Q_{1}, Q_{2}\right](c) \in B(b)_{c}$, for if $Q_{1}^{\prime}, Q_{2}^{\prime}$ are their restrictions to $B(b)$ then Lemma 3 shows $Q_{1}^{\prime}(d), Q_{2}^{\prime}(d)$ $\in B(b)_{d}$ for all $d \in B(b)$, hence $\left[Q_{1}, Q_{2}\right](c)=\left[Q_{1}^{\prime}, Q_{2}^{\prime}\right](c) \in B(b)_{c}$. This proves $\Delta_{c} \subseteq B(b)_{c}$ for all $c \in B(b)$.

Thus $\Delta$ is a distribution on the manifold $B(b)$. We prove now that $\Delta$ is involutive and has a differentiable base in the neighborhood of any point of $B(b)$. Let $Q_{1}, \cdots, Q_{r}$ be any base for $\mathcal{L}(b)$ and $K_{1}, \cdots, K_{d}$ any differentiable base for $H$ in the neighborhood of any $c \in B(b)$. Let $Q_{i}^{\prime}$ and $K_{i}^{\prime}$ denote the restrictions of $Q_{i}$ and $K_{i}$ to $B(b)$. The $Q_{i}^{\prime}$ and $K_{i}^{\prime}$ are clearly a differentiable base for $\Delta$ throughout a neighborhood of $c$ and to show $\Delta$ involutive it is sufficient, by Proposition 1, p. 88 of [6] to show the bracket of any pair of elements from such a base belongs to $\Delta$. But $\left[Q_{i}^{\prime}, Q_{j}^{\prime}\right]$ belongs to $\Delta$ because $\mathcal{L}(b)$ is closed under brackets, $\left[K_{i}^{\prime}, K_{j}^{\prime}\right]$ belongs to $\Delta$ from the definition of $\Delta_{d}$, and $\left[Q_{i}^{\prime}, K_{j}^{\prime}\right]$ belongs to $\Delta$ because Lemma 1 of $\$ 1$ implies it is horizontal.

Now we show $\Delta_{c} \supseteq B\left(b_{c}\right)$, hence $\Delta_{c}=B(b)_{c}$, for all $c \in B(b)$. Let $\rho$ be a piecewise differentiable horizontal curve in $B(b)$ from $b$ to $c$. Because it is horizontal, $\Delta$ is involutive, and $\Delta_{d} \supseteq H_{d}$, we can prove $\rho$ lies in the integral manifold $D$ of $\Delta$ through $b$. This can be done by extending the tangent vectors to $\rho$ to a differentiable horizontal vector field $K$ on a neighborhood of $b$; then $K$, when restricted to $D$, must have an integral curve through $b$ in $B$ and by uniqueness of integral curves in $B(b)$ this must be $\rho$. This shows $D \supseteq B(b)$, hence $\Delta_{c} \supseteq B(b)_{c}$.

Now note, for any $c \in B(b)$, that if $s$ and $t$ are in $H_{c}$ and $K_{1}, K_{2}$ are any horizontal differentiable vector fields on a neighborhood of $c$ with $K_{1}(c)=s$, $K_{2}(c)=t$, then $\Omega(s, t)=-\omega\left(\left[K_{1}, K_{2}\right](c)\right)$. This holds because the formula for $\Omega=D \omega$ as the "horizontal part" of the exterior derivative gives, for any vector fields $W_{1}, W_{2}$ on $B$ and linear functional $f$ on $L$,

$$
\begin{aligned}
f\left(D \omega\left(W_{1}(c), W_{2}(c)\right)\right)= & \left(H W_{1}(c)\right)\left((f \circ \omega)\left(H W_{2}\right)\right)-\left(H W_{2}(c)\right)\left((f \circ \omega)\left(H W_{1}\right)\right) \\
& -(f \circ \omega)\left(\left[H W_{1}, H W_{2}\right](c)\right) .
\end{aligned}
$$

Because $\omega$ is vertical, i.e. vanishes on all horizontal vectors, this reduces to

$$
f\left(D \omega\left(W_{1}(c), W_{2}(c)\right)\right)=-(f \circ \omega)\left(\left[H W_{1}, H W_{2}\right](c)\right)
$$

and hence

$$
D \omega\left(W_{1}(c), W_{2}(c)\right)=-\omega\left(\left[H W_{1}, H W_{2}\right](c)\right) .
$$

This trivially implies the above statement. Hence, for any fixed $c \in B$, the set of all such $\Omega(s, t)$ is the set of all $\omega\left(V\left[K_{1}, K_{2}\right](c)\right)$. Thus the set $R$ of all $\Omega(s, t)$ where $s$ and $t$ run through $B(b)_{c}$ and $c$ runs over $B(b)$ is the set of all $\omega\left(V\left[K_{1}, K_{2}\right](c)\right)$ where $K_{1}$ and $K_{2}$ run through all horizontal vector fields defined on a $B$-neighborhood of $c$ and $c$ runs over $B(b)$. The set of all these $V\left[K_{1}, K_{2}\right](c)$ is the same as the set of all $Q(c)$ where $Q \in R$ and $c \in B(b)$ and, 
since $\omega(Q(c))=\omega(Q(d))$, Lemma 3 implies $R$ is the set of all $\omega(Q(b))$ (b fixed). Thus $q(R)=\mathbb{R}$ and hence $q(L(b))=\mathcal{L}(b)$. Our definition of the differentiable structure on $B(b)$ shows, for $\phi$ any strip map taking $(m, e) \rightarrow b(m=\pi b)$, that $\phi_{m}$ takes the set of all $A(e)$, where $A$ is in the Lie algebra of $G(b)^{0}$, onto the vertical part of $B(b)$. Hence, by what was proved above, $\phi_{m}$ takes the set of all such $A(e)$ onto the vertical part of $\Delta_{b}$, i.e. onto the set of all $Q(b)$ where $Q \in \mathcal{L}(b)$. Because $q(L(b))=\mathcal{L}(b)$ this shows that $L(b)$ is the Lie algebra of $G(b)^{0}$. Theorem 2 now follows from Lemma 4.

COROLlaRY. If $L^{\prime}$ is the subalgebra of $L$ generated by all $\Omega(s, t)$ where $s$ and $t$ run through all pairs of tangent vectors at all points of $B$, then the subgroup of $G$ generated by $L^{\prime}$ is the component of the identity of the normal subgroup of $G$ generated by all the $G(b)$ for $b \in B$.

\section{BIBLIOGRAPHY}

1. A. Borel and A. Lichnerowicz, Groupes d'holonomie des variettés riemannienes, C.R. Acad Sci. Paris vol. 234 (1952) pp. 1835-1837.

2. E. Cartan, Les groupes d'holonomie des espaces généralises, Acta Math. vol. 48, pp. 1-42.

3. H. Cartan, Notions d'algebre differentielle; application aux groupes de Lie et aux variétés oì opère un groupe de Lie, Colloque de topologie, Bruxelles, 1950, pp. 15-27.

4. - La transgression dans un groupe de Lie et dans un espace fibre principal, Colloque de topologie, Bruxelles, 1950, pp. 57-71.

5. S. S. Chern, Notes on differential geometry, Institute for Advanced Study notes, 1951.

6. C. Chevalley, Lie groups, Princeton University Press, 1946.

7. C. Ehresmann, Les connexions infinitesimales dans un espace fibre différentiable, Colloque de topologie, Bruxelles, 1950, pp. 29-55.

8. H. Yamabe, On an arcwise connected subgroup of a Lie group, Osaka Math. J. vol. 2 (1950) pp. 13, 14 .

Massachusetts Institute of Technology,

Cambridge, Mass. 